Lamkaddem, M., Spreeuwenberg, P.M., Devillé, W.L., Foets, M.M., Groenewegen, P.P. Importance of quality
aspects of GP care among ethnic minorities: role of cultural attitudes, language and healthcare system of
reference. Scandinavian Journal of Public Health: 2012, 40(1), 25-34
\begin{tabular}{|c|l|}
\hline Postprint Version & 1.0 \\
\hline Journal website & $\underline{\text { http://sjp.sagepub.com/content/early/2011/10/15/1403494811425710.abstract }}$ \\
\hline Pubmed link & $\underline{\text { http://WwW.ncbi.nlm.nih.gov/pubmed/22013158 }}$ \\
\hline DOI & $10.1177 / 1403494811425710$ \\
\hline
\end{tabular}

This is a NIVEL certified Post Print, more info at http://www.nivel.eu

\title{
Importance of quality aspects of GP care among ethnic minorities: Role of cultural attitudes, language and healthcare system of reference.
}

\author{
MAJDA LAMKADDEM ${ }^{1,2}$, PETER M. SPREEUWENBERG ${ }^{1}$, WALTER L. DEVILLÉ ${ }^{\prime 1,3}$, MARLEEN \\ M. FOETS ${ }^{4} \&$ PETER P. GROENEWEGEN ${ }^{1,5,6}$ \\ ${ }^{1}$ Netherlands Institute for Health Services Research, Utrecht, The Netherlands, \\ ${ }^{2}$ Department of Public Health, University of Amsterdam, Amsterdam, The Netherlands, \\ ${ }^{3}$ Medical Anthropology and Sociology Unit, University of Amsterdam, Amsterdam, The Netherlands, \\ ${ }^{4}$ Institute of Health Policy and Management, Erasmus University, Rotterdam, The Netherlands,. \\ ${ }^{5}$ Department of Sociology, University of Utrecht, Utrecht, The Netherlands, and \\ ${ }^{6}$ Department of Human Geography, University of Utrecht, Utrecht, The Netherlands.
}

\begin{abstract}
.
Aims: This study examines the mechanisms responsible for ethnic differences in perceived quality of care in the Netherlands.

The specific role of cultural attitudes, language proficiency, and the health system in the country of origin was examined, taking socio-demographic characteristics into account. Methods: Interview data of 1339 respondents of Moroccan, Turkish, Surinamese and Antillean origin were combined with interview data of Dutch respondents $(n=405)$ and of Western immigrants $(\mathrm{n}=102)$ in the Netherlands and of a random sample of Dutch privately or publicly insured persons $(n=9675)$.

Data collection took place within the Second Dutch National Survey of General Practice (DNSGP-2, 2001). Items from the QUality Of care Through the patient's Eyes (QUOTE) questionnaire were used to measure expectations, as well as items from the QUOTE-Mi (adapted version for migrant groups). Items on normative orientations were used to measure cultural attitudes. Results: In contrast to our hypothesis, respondents with more egalitarian/modern attitudes attached less importance to quality aspects related to access and quality. Tests on the role of the health system of reference were generally conclusive, showing that respondents accustomed to (parts of) another system have different expectations regarding several aspects of general practitioner healthcare quality, e.g. access to specialist care. Conclusions: Besides socio-demographic characteristics, culture influences patients' expectations regarding general practitioner care quality.

However, the role of culture can be more clearly ascribed to the characteristics of the health system which is held as the reference than to the general attitudes on normative orientations.
\end{abstract}

\section{INTRODUCTION.}

Patients' views on quality of care can have a considerable impact on health outcomes. A low perceived quality of care may lead to non-adherence to medical prescriptions [1], or inadequate attendance to healthcare providers [2-4], potentially leading to negative health outcomes. Ethnic differences in perceived quality of care are well documented, but few studies have examined how these differences arise [5-8]. 
Lamkaddem, M., Spreeuwenberg, P.M., Devillé, W.L., Foets, M.M., Groenewegen, P.P. Importance of quality
aspects of GP care among ethnic minorities: role of cultural attitudes, language and healthcare system of
reference. Scandinavian Journal of Public Health: $2012,40(1), 25-34$

Adjustment for socioeconomic characteristics only partly explains these differences in perceived hajudiefy of care [8-11].

The present study examines which mechanisms are responsible for ethnic differences in perceived quality of care in the Netherlands. More specifically, the research question is: to what extent does culture contribute to the understanding of ethnic differences in perceived quality of general practitioner care? As perceptions are closely related to culture, we examined the influence of cultural components on the importance ratings of several aspects of quality of care. Differentiating between the ratings of actual performance and the importance ratings offers insight into ethnic differences in expectations.

Two culture-related hypotheses on ethnic differences in the importance given to several aspects of general practitioner (GP) healthcare quality are explored. The first hypothesis is related to the attitudes and language of a cultural group, the second concerns the institutions shared by a group.

This second hypothesis is tested through three implications.

\section{Hypothesis 1: cultural attitudes and language.}

It is generally accepted that culture not only concerns ways of acting, but also ways of thinking/feeling that are common (learned and shared) to a group and distinguish them as such. Those components of culture are traditionally assessed at the individual level by focusing on, for example, language proficiency and attitudes concerning (amongst others) religion, gender roles, and authority. These attitudes are assumed to influence the way quality of care is perceived. Modernisation theories share the assumption that industrialisation causes changes in topics such as gender roles and attitudes towards authority [12], leading to more interchangeable gender roles and egalitarian views.

Therefore, we hypothesised that more modern attitudes that are closer to mainstream Dutch attitudes and values (i.e. secularised, more egalitarian views of gender roles, and rejection of traditional authority) would coincide with higher ratings of aspects of healthcare quality that are related to choice and selfdetermination. Similarly, it was hypothesised that greater proficiency in the Dutch language would be associated with higher ratings of the same quality aspects, because of its direct influence on patient empowerment [13] and, therefore, on assertiveness in the relationship with healthcare providers. Thus, we tested: Hypothesis 1A: respondents from ethnic minorities with more modern attitudes give a higher rating to aspects of healthcare quality that are related to choice and self-determination; and Hypothesis 1B: greater proficiency in the Dutch language is associated with higher ratings of these quality aspects.

Hypothesis 1 was tested in a sample of respondents of Turkish, Moroccan, Surinamese, and Antillean origins (the four main ethnic minorities in the Netherlands).

\section{Hypothesis 2: shared institutions and healthcare system of reference.}

National groups share a common experience regarding the functioning of institutions such as the healthcare system. Being in contact with the institutions of a society, an individual becomes accustomed to their patterns of conduct [14]. In case of migration, the previously learned patterns of conduct may remain the first reference from which the new healthcare system is judged. Therefore, we hypothesised that people develop the criteria to judge the quality of health care in the course of their socialisation, and that they use the health system they grew up in as their (system of) reference.

We examined three different implications of the shared institutions hypothesis. The first implication is that the role of the system of reference may be reinforced by use of healthcare services in the country of origin. This may occur among those who regularly visit their country of origin for long vacations, for example. It is reported that migrants who live transnationally become members of more than one society and therefore health system, in which they place trust and build expectations [15]. We tested implication A among respondents of Turkish and Moroccan origin living in the Netherlands. These two groups were selected because (i) many of them regularly visit their country of origin for vacations and maintain contact with it, and (ii) an important difference between the health systems in Turkey and Morocco and the Netherlands is the direct access to specialist health care in Turkey and Morocco versus the gatekeeping system in the Netherlands. Thus we tested Hypothesis 2A: respondents who regularly use the healthcare system in the country of origin would value some of its properties more than those who do not (especially the larger accessibility of specialist care).

The second implication concerns immigrants of Western origin in the Netherlands. The gatekeeping system is not common in other Western countries.

Most Western immigrants in the Netherlands are of German or Belgian origin, countries with direct access to specialist care. Therefore, we assumed that migrants of Western origin would rate the importance of access criteria to secondary care higher than Dutch people who are not used to such a system. Thus we 


\begin{abstract}
Lamkaddem, M., Spreeuwenberg, P.M., Devillé, W.L., Foets, M.M., Groenewegen, P.P. Importance of quality aspects of GP care among ethnic minorities: role of cultural attitudes, language and healthcare system of reference. Scandinavian Journal of Public Health: 2012, 40(1), 25-34

tested Hypothesis 2B: Western immigrants rate aspects of healthcare quality related to accessibility $n j$ le specialist care higher than Dutch people.

Finally, the third implication concerns differences between people in the Netherlands who were privately insured versus those publicly insured (statutory health insurance). In the former health insurance system (still in place at the time of the data collection until 2006 [16]), people with a private insurance were enjoying a (slightly) broader choice in access to health services than publicly insured persons $[17,18]$. Therefore, in the line of the general hypothesis, we assumed that privately insured people would rate access/service factors as more important than do publicly insured respondents. Thus we tested Hypothesis 2C: privately insured people rate aspects of healthcare quality that are related to accessibility of specialist care higher than publicly insured people.
\end{abstract}

\title{
METHODS.
}

\section{Data collection.}

Data collection took place within the Second Dutch National Survey of General Practice (DNSGP-2), carried out in 2001. That study was conducted within a representative sample of 104 general practices located throughout the Netherlands. The data include background information on patients collected via a census, approximately 12000 health interview surveys and more than 1 million recorded contacts of patients with their GPs in both years [19].

Within the DNSGP-2, face-to-face interviews were conducted among a sample of 1339 participants from the four main minority groups in the Netherlands, i.e. (children of) immigrants from Turkey, Morocco, Surinam, and the Dutch Antilles.

The questionnaire was translated into the native languages (forward-backward method) and field tested. Respondents were interviewed in the language of their choice. Interviewers and respondents were matched on ethnicity. The response rate was about $50 \%$, with no significant difference in response between the four groups. The main reasons for nonresponse were that respondents could not be reached (24.9\%) or that they declined to participate (19.5\%). Small differences existed between ethnic groups as to the reason for nonresponse, with Antillean and Surinamese nonrespondents more likely to be unreachable $(53.3 \%$ and $54.3 \%$ vs. refusal, respectively) than Turkish and Moroccan nonrespondents ( $43.9 \%$ and $48 \%$, respectively).

These interview data were used to perform analyses on the first hypothesis and the second hypothesis (implication A).

Interview data from a random sample from the DNSGP-2 of Dutch respondents and of non-Dutch respondents of Western origin were used to test the second hypothesis (implication $B)$. This sample $(n=507)$ included about four times as many Dutch people $(n=405)$ than the total number of migrants of Western origin ( $\mathrm{n}=102)$, for statistical comparison purposes [20]. Western immigrants came mostly from neighbouring countries such as Germany $(n=23)$ and Belgium $(n=18)$. All the Western immigrants were sufficiently fluent in the Dutch language to be interviewed.

Implication $C$ of the second hypothesis (implication C) was also tested among a DNSGP-2 sample $(n=9675)$ of Dutch privately insured $(n=3305)$ and publicly insured persons $(n=6370)$ (Western immigrants excluded).

All respondents were aged 18 years and older, and the study population included GP attenders and nonattenders [19]. Table I summarises the use of the samples for the different hypotheses.

\section{Measures.}

Perceived quality of care: QUOTE and QUOTEMi Perceived quality of care was measured with the general QUality Of care Through the patient's Eyes (QUOTE) questionnaire and with the migrant version of the questionnaire: QUOTE-Mi. The QUOTE was developed to measure quality of care from the patient's perspective based on 22 items (Table II), each concerning a specific aspect of the GP health care [21]. An adapted version for immigrants, the QUOTE-Mi, was developed earlier [22].

The QUOTE instruments (general and migrant specific) combine importance ratings and actual performance ratings for each item. The importance ratings are measured on a 4-point scale, ranging from "not important" to "extremely important". Data on actual performance were not used.

The version of the QUOTE-Mi used in these analyses is based on 15 items. These items have a scale structure (with five dimensions) based on factor and reliability analyses (Table II). The five scales were access, equity, treatment, language, and ethnic identity (or ethnicity). Access refers to the general accessibility of the GP practice and referrals to other healthcare providers. Equity refers to the extent to 
Lamkaddem, M., Spreeuwenberg, P.M., Devillé, W.L., Foets, M.M., Groenewegen, P.P. Importance of quality
aspects of GP care among ethnic minorities: role of cultural attitudes, language and healthcare system of reference. Scandinavian Journal of Public Health: 2012, 40(1), 25-34 which patients feel treated equally, regardless of their ethnic background or other specificities. Treativent concerns the information process regarding medical treatment. Language refers to all matters concerning language differences and the way to bridge communication gaps (information folders, use of an interpreter, etc.). Finally, Ethnic identity concerns the GP's recognition of the ethnic and cultural specificity of the patient (Table II). Scales were computed by summing the scores of all items of the scale and dividing the total by the number of items in each scale. Missing cases were excluded listwise (on average $2.9 \%$ case loss per scale).

Cultural attitudes and language We made use of items from the normative orientations part of the DNSGP- 2 interview with the ethnic minority groups. Besides language proficiency, we had information on the following culture-related concepts [12]: perception of gender roles exchangeability, secularisation and authority (rejection). Table II shows the items used to measure these four cultural dimensions. Scales were computed by summing the scores of all items of the scale and dividing the total by the number of items in each scale. Missing cases were excluded listwise, because of the small cases loss (on average $0.19 \%$ per scale).

Language proficiency was assessed by means of self-report on the ability to understand, speak, read and write in Dutch (four items, each using a 4-point scale ranging from "'not" to ' good"', summarised into one scale by calculating averages).

\section{[TABLE 1] [TABLE 2].}

\section{Statistical analyses.}

Multilevel analysis was used for the regression models. Because patients were hierarchically nested within GP practices this could theoretically influence the estimates of the dependent variables (importance ratings scales on five quality aspects of GP care: access, equity, treatment, ethnicity, and language) and the standard errors of the coefficients of the independent variables. Regression coefficients were adjusted at both the individual (socio-demographic characteristics) and the GP practice (practice characteristics) level (confounders: age, gender, health status, education, insurance type, practice form, and level of urbanisation).

Models including only confounders (not shown) were compared to models summing the cultural and language indicators (explanatory variables) by testing the -2loglikelihood difference (deviance tests), in order to test the first hypothesis on cultural attitudes and language.

For the first implication (A) of the shared institutions hypothesis, we computed models comparing regression coefficients for the importance ratings for each QUOTE-Mi item (dependent variables) between respondents who did and did not have contact with a caregiver in the country of origin in the past year. These models were adjusted for the same confounders as the previous models at the individual and practice level, and subsequently for cultural attitudes and language proficiency.

For the second implication (B), we computed models comparing regression coefficients for the importance ratings of each QUOTE item (dependent variables) between respondents of Dutch origin and respondents from other Western countries. These models were adjusted for the same confounders as the previous models at both the individual and practice level.

For the third implication (C), we computed models comparing regression coefficients for the importance ratings of each QUOTE item (dependent variables) between respondents with a private and a public health insurance. These models were adjusted for the same confounders as the previous models at both the individual and practice level.

For implications A, B, and C, analyses were performed for all quality criteria included in the

\section{[TABLE 3].}

QUOTE and in the QUOTE-Mi, but only significant associations $(\mathrm{p}=0.05)$ with the variables of interest (healthcare use in the country of origin, Western immigrants vs. the Dutch, and insurance type) are presented in the tables. All analyses were performed using the statistical software package MLwiN 2.0. 


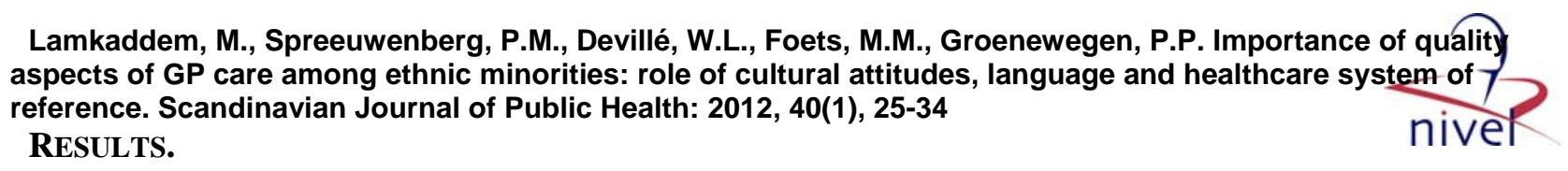

\section{Study population.}

DNSGP-2 data of 1178 respondents from ethnic minority groups were eligible (excluding cases with missing values) for analyses on the first hypothesis (quality of care and cultural attitudes/language) and implication A of the second hypothesis (shared institutions-healthcare use in the country of origin).

Of these, 24.7\% were of Moroccan, 20.5\% of Antillean, 31.4\% of Turkish, and 23.3\% were of Surinamese origin. The Moroccan group was on average the youngest (36 years), and the Surinamese the eldest (44 years). Proportionally more women took part in the study (58.7\%), and the overrepresentation was the largest in the Surinamese group, where $72 \%$ of all respondents were female.

Information on the study population of the additional dataset (Dutch and immigrants of Western origin) are published elsewhere [19].

Quality of care and cultural attitudes/language Compared to the models including only sociodemographic and practice characteristics (not shown), all models significantly improved $(\mathrm{p}<0.001)$ their explanatory power when adding information on the four cultural and language indicators, except for the ethnicity dimension. In that case, adding cultural variables to the models did not significantly improve the importance models with confounders. This implies that taking cultural attitudes and language proficiency into account (besides socioeconomic status) generally improves our understanding of ethnic variation in what respondents consider important in GP health care.

Ethnic differences in importance ratings When comparing importance ratings between the four groups in the final models, ethnic differences are still substantial, even after adjusting for the four cultural variables and the several confounders (Table III). On the whole, the ratings of the Turkish group were the highest significantly higher than the other three groups on the access, equity, and language dimensions, and higher than the Moroccans on the treatment dimension) - but not for the dimension of ethnicity, where the ratings of the Turkish and Moroccan respondents were similar and both significantly higher than the ratings of the Antillean and the Surinamese participants.

Cultural attitudes and language Language proficiency significantly predicted importance ratings on the access, equity, and language dimensions of the QUOTE-Mi. Secularisation was significantly associated with all dimensions except ethnicity. Authority rejection was significantly associated with importance ratings on access, equity, and treatment dimensions.

Gender roles exchangeability significantly explained ratings on the treatment dimension.

Finally, the ethnicity dimension was not associated with any cultural indicator (Table III).

Significant associations between importance ratings and cultural factors were often negative: i.e.

respondents with more modern cultural attitudes rated quality dimensions as less important. Only language proficiency was positively related to the importance of access and equity dimensions: i.e.

people with greater language proficiency attached more importance to these quality aspects. Greater language proficiency was also associated with lower

\section{[TABLE 4].}

ratings of quality items such as involving an interpreter and providing leaflets in one's own language. Another exception was the positive association between gender roles exchangeability and the treatment dimension, indicating that higher importance given to information on medical treatment was associated with a more egalitarian view of gender roles. However, this association was only marginally significant. The negative direction of the other significant associations indicated (amongst others) that a less secularised vision was related to a higher importance given to quality aspects such as access, equity, treatment, and language. Similarly, respondents with a broader acceptance of authority rated quality aspects on access, equity, and treatment as more important. The direction of those associations is opposite to the formulated hypothesis, which related more egalitarian/modern views to a more demanding attitude on the quality of GP health care.

\section{Shared institutions.}

Healthcare use in country of origin (hypothesis 2A) In the Moroccan group, 159 of the 291 respondents went to Morocco during their last vacation: of those, $22.2 \%$ had contact with a local healthcare provider. A similar proportion (55.7\%) of the Turkish group spent their last vacation in Turkey and, of those, 29.1\% had contact with a local healthcare provider.

Table IV presents the regression coefficients between quality importance ratings (dependent variables) and use of health care in the country of origin (predictor): only significant coefficients for at least one of both 


\begin{abstract}
Lamkaddem, M., Spreeuwenberg, P.M., Devillé, W.L., Foets, M.M., Groenewegen, P.P. Importance of quality aspects of GP care among ethnic minorities: role of cultural attitudes, language and healthcare system of reference. Scandinavian Journal of Public Health: 2012, 40(1), 25-34

groups are shown. Moroccan respondents who had contact with a healthcare provider in Morocco inithel past year considered the four (of the 15 items of the QUOTE-Mi) aspects as more important than those who did not. This was in no case significant for the Turkish group. In line with the hypothesis, the significant associations mostly concerned the access dimension (in three cases, out of the five items constituting the scale). The other item ('Recognise that my problems might differ from those of Dutch people') constitutes one of the two items included in the ethnic identity dimension.

Dutch vs. Western immigrants (hypothesis $2 B$ ) Table $\mathrm{V}$ presents the significant associations between quality importance ratings (dependent variables) and origin (Dutch or Western immigrant).

Western foreigners considered a (quick) referral to specialist care more important than the Dutch population did. On the other hand, Dutch
\end{abstract}

\title{
[TABLE 4].
}

respondents considered matters such as accessibility of the GP by telephone and that the GP complies strictly with his/her appointments as more important than the Western foreigners (negative associations between importance scores and being a Western immigrant). The rest of the 22 items of the QUOTE showed no significant differences between the indigenous Dutch and Western immigrants.

Public vs. private insurance (hypothesis 2C) In the general population sample, $65.8 \%$ was insured publicly and the remainder had private insurance. Table VI presents the significant associations between importance ratings (dependent variables) and type of insurance. Eight of the 22 QUOTE quality importance ratings were significantly associated with insurance status.

In six of the eight cases, privately insured respondents rated the quality aspect concerned as more important than the publicly insured. The two associations taking the reverse direction were the prescription of medication refunded by the insurance company and the readiness of the GP to talk about problems.

\section{DISCUSSION.}

The present study examined which factors (besides socioeconomic differences) are responsible for ethnic differences in perceived quality of care in the Netherlands. To our knowledge, few studies have examined the mechanisms involved in ethnic differences in patients' expectations [8, 23], even though these can lead to differences in perceived quality of care [23] and to inequities in health care [3]. Our hypotheses focused on the role of "modern" cultural attitudes on the one hand and of the healthcare system of reference (i.e. the one of the country of origin for migrants having regular contact with its providers) on the other.

\section{Language proficiency.}

Our hypothesis linking proficiency in the Dutch language with higher ratings of quality aspects, such as access and treatment, was confirmed by the analyses. As expected, those with greater language proficiency also rated items related to translation services in the GP practice as less important, probably because of their limited need for such services.

Are "modern" patients more demanding? The fact that (in general) egalitarian/modern attitudes were related to less importance of most quality aspects among the selected ethnic minorities was unexpected, based on the assumption that patients' emancipation (theoretically linked to values such as secularisation and rejection of traditional authority [12]) would be responsible for more demanding requirements on patients' behalf. However, this unexpected result leaves the second hypothesis intact, because the higher/more demanding expectations of patients with less egalitarian/modern attitudes could result from a closer bond with the healthcare system in the country of origin and its norms for those showing less mainstream Western attitudes.

\section{Does another health system constitute the reference?.}

The first implication of the health system of reference hypothesis is about differences in ratings between respondents who did or did not have contact with a healthcare provider in the country of origin in the past year. Those analyses took cultural attitudes and language proficiency into account and showed important differences between those two groups for the Moroccan respondents. This implies that, for the Moroccans, besides cultural variables, having (ongoing) contact with the healthcare system of the country of origin influences the way aspects of the Dutch healthcare system are perceived as important. In the Funding. This work was supported by the Netherlands Organization for Scientific Research, Social Cohesion Program; subprogram, the Dutch Multicultural and Pluriform Society (MPS) (grant number 26198618). 
Lamkaddem, M., Spreeuwenberg, P.M., Devillé, W.L., Foets, M.M., Groenewegen, P.P. Importance of quality aspects of GP care among ethnic minorities: role of cultural attitudes, language and healthcare system of reference. Scandinavian Journal of Public Health: 2012, 40(1), 25-34

REFERENCES.

[1] Wroth TH, Pathman DE. Primary medication adherence in a rural population: the role of the patientphysician relationship and satisfaction with care. J Am Board Fam Med 2006;19(5):478-86.

[2] Neal RD, Heywood PL, Morley S. "I always seem to be there" - a qualitative study of frequent attenders. Br J Gen Pract 2000;50(458):716-23.

[3] Kersnik J, Svab I, Vegnuti M. Frequent attenders in general practice: quality of life, patient satisfaction, use of medical services and GP characteristics. Scand J Prim Health Care 2001;19(3):174-7.

[4] Martin C, Perfect T, Mantle G. Non-attendance in primary care: the views of patients and practices on its causes, impact and solutions. Fam Pract 2005;22(6):638-43.

[5] Henry J. Kaiser Family Foundation. Race, ethnicity and family care: a survey of public perceptions and experiences.

Menlo Park: Henry J. Kaiser Foundation, 1999.

[6] Fiscella K, Franks P, Gold MR, Clancy CM. Inequality in quality. Addressing socioeconomic, racial and ethnic disparities in health care. JAMA 2000;283(19):2579-84.

[7] Boulware LE, Cooper LA, Ratner LE, LaVeist TA, Powe NR. Race and trust in the health care system. Public Health Rep 2003;118(4):358-65.

[8] Uiters E. Primary health care use among ethnic minorities in The Netherlands: a comparative study [dissertation].

Utrecht. NIVEL, 2007.

[9] Hunt KA, Gaba A, Lavizzo-Mourey R. Racial and ethnic disparities and perceptions of health care: does health plan type matter? Health Serv Res 2005;40(2):551-76.

[10] Haviland MG, Morales LS, Dial TH, Pincus HA. Race/ ethnicity, socioeconomic status and satisfaction with health care. Am J Med Qual 2005;20(4):195-203.

[11] Taira DA, Gelb Safran D, Seto TB, Rogers WH, Inui TS, Montgomery J, et al. Do patient assessments of primary care differ by patient ethnicity? Health Serv Res 2001;36(6 Pt 1):1059-71.

[12] Inglehart R, Baker WE. Modernization, cultural change and the persistence of traditional values. Am Sociol Rev 2000;65(1):19-51.

[13] Garrett PW, Dickson HG, Lis-Young,Whelan AK, Roberto- Forero. What do non-English-speaking patients value in acute care? Cultural competency from the patient's perspective: a qualitative study. Ethn Health 2008;13(5):479-96.

[14] Berger PL, Luckmann T. The social construction of reality: a treatise in the sociology of knowledge. Garden City (NY): Anchor Books, 1966.

[15] Bryceson D, Vuorela U. The transnational family. New European frontiers and global networks. Oxford: Berg, 2002.

[16] Bartholome'e Y, Maarse H. Health insurance reform in the Netherlands. Eurohealth 2006;12(2):7-9.

[17] Bongers IM, van der Meer JB, van den Bos J, Mackenbach JP. Socio-economic differences in general practitioner and outpatient specialist care in The Netherlands: a matter of health insurance? Soc Sci Med 1997;44(8):1161-8.

[18] Tapay N, Colombo F. Private health insurance in the Netherlands: a case study. Paris: OECD, 2004.

[19] Westert GP, Schellevis FG, de Bakker DH, Groenewegen PP, Bensing JM, van der Zee J. Monitoring health inequalities through general practice: the Second Dutch National Survey of General Practice. Eur J Public Health 2005;15(1):59-65.

[20] Belle G van. Statistical rules of thumb. New York: Wiley, 2004.

[21] Sixma HJ, Kerssens JJ, van Campen C, Peters L. Quality of care from the patients' perspective: from theoretical concept to a new measuring instrument. Health Expect 1998;1(2):82-95.

[22] El Fakiri F, Sixma HJ, Weide MG. Kwaliteit van de huisartsenzorg vanuit migrantenperspectief: ontwikkeling van een meetinstrument [Quality of general practitioner care from a migrant's viewpoint: development of a measurement instrument]. Utrecht: NIVEL, 2000.

[23] Campbell JL, Ramsay J, Green J. Age, gender, socioeconomic, and ethnic differences in patients' assessments of primary health care. Qual Health Care 2001;10(2):90-5.

[24] Lamkaddem M, Spreeuwenberg P, Deville' W, Foets M, Groenewegen P. Changes in health and primary health care use of Moroccan and Turkish migrants between 2001 and 2005: a longitudinal study. BMC Public Health 2008;8:40-8.

TABLE 
Lamkaddem, M., Spreeuwenberg, P.M., Devillé, W.L., Foets, M.M., Groenewegen, P.P. Importance of quality aspects of GP care among ethnic minorities: role of cultural attitudes, language and healthcare system of reference. Scandinavian Journal of Public Health: 2012, 40(1), 25-34

Table I. Methods summary: samples and analyses

\begin{tabular}{|c|c|c|c|}
\hline Hypothesis/implication & Sample from DNSGP-2 & Sample composition & Dependent variables in models \\
\hline \multirow[t]{4}{*}{1} & $\mathrm{~A}(n=1339)$ & $\operatorname{Turkish}(n=405)$ & $\begin{array}{l}\text { Importance sumscores scales } \\
\text { QUOTE-Mi }\end{array}$ \\
\hline & & $\operatorname{Moroccan}(n=373)$ & \\
\hline & & Surinamese $(n=298)$ & \\
\hline & & Antillean $(n=263)$ & \\
\hline \multirow[t]{2}{*}{ 2/A } & Subsample of A $(n=438)$ & Turkish $(n=232)$ & $\begin{array}{l}\text { Importance ratings items } \\
\text { QUOTE-Mi }\end{array}$ \\
\hline & $\begin{array}{l}\text { Selection criterion: Turkish/ } \\
\text { Moroccan and spent the last } \\
\text { holiday in country of origin }\end{array}$ & Moroccan $(n=206)$ & \\
\hline $2 / \mathrm{B}$ & $\mathrm{B}(n=507)$ & $\begin{array}{l}\operatorname{Dutch}(n=405) \\
\text { Western migrants }(n=102)\end{array}$ & Importance ratings items QUOTE \\
\hline $2 / \mathrm{C}$ & $\mathrm{C}(n=9675)$ & $\begin{array}{l}\text { Dutch privately insured }(n=3305) \\
\text { Dutch publicly insured }(n=6370)\end{array}$ & Importance ratings items QUOTE \\
\hline
\end{tabular}

QUOTE, QUality Of care Through the patient's Eyes questionnaire; QUOTE-Mi, QUOTE adapted version for migrant groups.

Table II. Measures

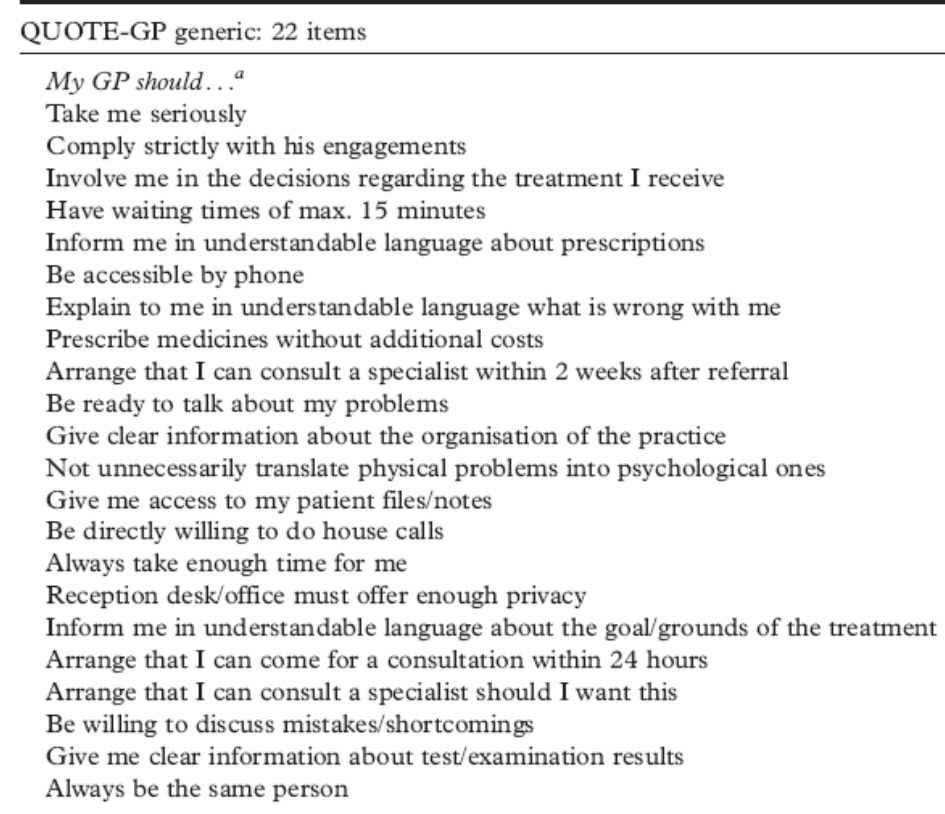

QUOTE-Mi: 15 items

Factor loadings after

My GP should... ${ }^{a}$

Access

Arrange that I can consult a specialist within 2 weeks after referral

Varimax rotation

0.759

0.736

Give clear information about the organisation of the practice 0.704

$\begin{array}{ll}\text { Arrange that I can come for a consultation within } 24 \text { hours } & 0.657\end{array}$

Language

Involve me in the decisions regarding the treatment I receive $\quad 0.420$

$\begin{array}{ll}\text { Involve an interpreter should I want this } & 0.835\end{array}$

Have information leaflets in my own language $\quad 0.757$

$\begin{array}{ll}\text { Understand that I sometimes have difficulties in } & 0.740\end{array}$

Equity

expressing myself due to language problems

$\begin{array}{ll}\text { Give me as much time and attention as Dutch patients } & 0.808 \\ \text { Take me seriously } & 0.725\end{array}$

$\begin{array}{ll}\text { Not have prejudices about my being a foreigner } & 0.7256\end{array}$ 
Lamkaddem, M., Spreeuwenberg, P.M., Devillé, W.L., Foets, M.M., Groenewegen, P.P. Importance of quality aspects of GP care among ethnic minorities: role of cultural attitudes, language and healthcare system of 7 reference. Scandinavian Journal of Public Health: 2012, 40(1), 25-34

Treatment

Inform me in understandable language about prescriptions $\quad 0.833$

Ethnic identity

Explain to me in understandable language what is wrong with me $\quad 0.774$

Show interest in my cultural background $\quad 0.830$

Recognise that my problems might differ from those of Dutch people $\quad 0.644$

Cultural components

Secularisation $^{b}$

It is a pity that religion is losing importance in the Netherlands

(Dutch) language proficiency

Able to understand Dutch?

Able to speak Dutch?

Able to read Dutch?

Authority (rejection)

Able to write Dutch?

Gender role division

In the Netherlands there is too little respect for authority figures like doctors, teachers, and policemen

The man should make decisions concerning large purchases

The woman should make decisions concerning housekeeping

The man should make decisions about money matters

An education is more important for boys than for girls

It is more important for boys to have their own income than for girls

A woman should stop working when she has children

Answer categories: ${ }^{a}$ not important/fairly important/important/extremely important; ${ }^{b}$ completely agree/agree/neither agree nor disagree/ disagree/completely disagree; ${ }^{c}$ not/a little/sufficiently/well.

Table III. Average importance ratings and cultural indicators regression coefficents

\begin{tabular}{|c|c|c|c|c|c|}
\hline & Access & Equity & Treatment & Ethnicity & Language \\
\hline \multicolumn{6}{|l|}{ Adjusted group mean score $\mathrm{a}^{\mathrm{a}}$} \\
\hline Moroccan & $3.251(0.044)$ & $3.401(0.049)$ & $3.247(0.065)$ & $2.660(0.095)$ & $2.405(0.099)$ \\
\hline Antillean & $3.264(0.046)$ & $3.356(0.044)$ & $3.378(0.057)$ & $2.398(0.098)$ & $2.355(0.080)$ \\
\hline Turkish & $3.431(0.048)$ & $3.565(0.052)$ & $3.477(0.061)$ & $2.722(0.102)$ & $2.840(0.079)$ \\
\hline Surinamese & $3.202(0.057)$ & $3.389(0.058)$ & $3.400(0.065)$ & $2.277(0.113)$ & $2.204(0.093)$ \\
\hline Language proficiency & $0.069(0.019)$ & $0.059(0.022)$ & $0.018(0.027)$ & $0.049(0.039)$ & $-0.147(0.038)$ \\
\hline Secularisation & $-0.044(0.013)$ & $-0.040(0.014)$ & $-0.034(0.017)$ & $-0.037(0.026)$ & $-0.052(0.026)$ \\
\hline Authority rejection & $-0.063(0.013)$ & $-0.068(0.015)$ & $-0.089(0.018)$ & $-0.037(0.027)$ & $0.015(0.028)$ \\
\hline Gender roles exchangeability & $0.018(0.019)$ & $0.039(0.021)$ & $0.051(0.026)$ & $-0.035(0.039)$ & $0.010(0.039)$ \\
\hline
\end{tabular}

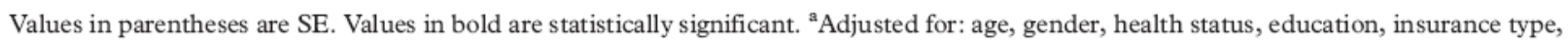
practice form, level of urbanisation, language proficiency, secularisation, authority rejection, gender roles exchangeability.

Table IV. Linear regression coefficients: contact with a caregiver in country of origin: no (0) vs. yes (1) (independent) (hypothesis 2A)

\begin{tabular}{lcc}
\hline My GP should ... (dependents) & Estimate & $p$-value \\
\hline Arrange that I can consult a specialist within 2 weeks after referral & & 0.032 \\
Moroccan & 0.249 & 0.525 \\
Turkish & 0.060 & 0.031 \\
Be accessible by phone & 0.206 & 0.543 \\
Moroccan & 0.047 & 0.010 \\
Turkish & 0.484 & 0.105 \\
Recognise that my problems might differ from those of Dutch people & 0.051 \\
Moroccan & 0.242 & 0.783 \\
Turkish & & 0.222 \\
Arrange that I can come for a consultation within 24 hours & 0.025 & \\
Moroccan & & \\
Turkish & & \\
\hline
\end{tabular}

Adjusted for: age, gender, health status, education, insurance type, practice form, level of urbanisation, language proficiency, secularisation, authority rejection, gender roles exchangeability. 
Lamkaddem, M., Spreeuwenberg, P.M., Devillé, W.L., Foets, M.M., Groenewegen, P.P. Importance of quality aspects of GP care among ethnic minorities: role of cultural attitudes, language and healthcare system of 7 reference. Scandinavian Journal of Public Health: 2012, 40(1), 25-34

$-: .$.

Table V. Linear regression coefficients: Dutch (0) vs. immigrants of Western origin (1) (independent) (hypothesis 2B)

\begin{tabular}{lcr}
\hline My GP should ... (dependents) & Estimate & $p$-value \\
\hline Comply strictly with his/her appointments & -0.173 & 0.024 \\
Be accessible by phone & -0.179 & 0.025 \\
Arrange that I can consult a specialist within 2 weeks after referral & 0.174 & 0.053 \\
\hline
\end{tabular}

Adjusted for: age, gender, health status, insurance type, education, practice form, level of urbanisation.

Table VI. Linear regression coefficients: public (0) vs. private (1) insurance type (independent) (hypothesis 2C)

\begin{tabular}{lcc}
\hline My GP should...(dependents) & Estimate & $p$-value \\
\hline Take me seriously & 0.044 & 0.000 \\
Comply strictly with his/her appointments & 0.055 & 0.000 \\
Involve me in the decisions regarding the treatment I receive & 0.042 & 0.014 \\
Having waiting times of max. 15 minutes & 0.070 & 0.002 \\
Prescribe medicines without additional costs & -0.229 & 0.000 \\
Be ready to talk about my problems & -0.047 & 0.031 \\
Give me access to my patient files/notes & 0.054 & 0.007 \\
Give me clear information about test/examination results & 0.031 & 0.010 \\
\hline
\end{tabular}

Adjusted for: age, gender, health status, education, practice form, level of urbanisation. 